\title{
Surgical Outcomes After Neoadjuvant Chemoradiation Followed by Curative Surgery in Patients With Esophageal Cancer
}

\section{An Intergroup Phase III Trial of the Swiss Group for Clinical Cancer Research (SAKK 75/08)}

\author{
Urs von Holzen, MD, MBA, FACS, ${ }^{*} \dagger$ Sven Schmidt, MD, $\ddagger$ Stefanie Hayoz, PhD, § Thomas Steffen, MD, $\uparrow$ \\ Felix Grieder, MD,\| Detlef Bartsch, MD, ${ }^{* *}$ Annelies Schnider, MD, †† Wolfram-Trudo Knoefel, MD, $\ddagger \ddagger$ \\ Guillaume Piessen, MD, $\S \S$ Christoph Kettelhack, MD, ${ }^{*}$ Walter R. Marti, MD, EMBA, $\uparrow \mid$ \\ Markus Schäfer, MD,|||| Reinhold Függer, MD, PhD, ${ }^{* * *}$ Alfred Königsrainer, MD, ${ }^{\dagger} \dagger \dagger$ \\ Beat Gloor, MD, 㧊 Markus Furrer, MD, §§§ Marie-Aline Gérard, PhD, § Hanne Hawle, MD, $\S$ \\ Martin K. Walz, MD, $\uparrow \uparrow \mid$ Piero Alesina, MD, $\uparrow \uparrow$ and Thomas Ruhstaller, MD||||||, for the Swiss Group for \\ Clinical Cancer Research (SAKK), the German Esophageal Cancer Study Group, the Austrian \\ Arbeitsgemeinschaft Medikamentöse Tumortherapie (AGMT), and the Fédération Francophone de Cancérologie \\ Digestive (FFCD)/Fédération de Recherche en Chirurgie (FRENCH)
}

Objective: To assess the impact of surgical technique in regard to morbidity and mortality after neoadjuvant treatment for esophageal cancer.

Background: The SAKK trial 75/08 was a multicenter phase III trial (NCT01107639) comparing induction chemotherapy followed by chemoradiation and surgery in patients with locally advanced esophageal cancer. Methods: Patients in the control arm received induction chemotherapy with cisplatin and docetaxel, followed by concomitant chemoradiation therapy with cisplatin, docetaxel, and $45 \mathrm{~Gy}$. In the experimental arm, the same regimen was used with addition of cetuximab. After completion of neoadjuvant treatment, patients underwent esophagectomy. The experimental

From the *University Hospital Basel, Basel, Switzerland Universitätsspital, Basel, Switzerland; †Indiana University School of Medicine South Bend, Goshen Center for Cancer Care, Goshen, Indiana; †Charité - University Medicine, Berlin, Germany now at Klinikum Ernst von Bergmann gemeinnützige $\mathrm{GmbH}$, Potsdam, Germany; §SAKK Coordinating Center, Bern, Switzerland; - Cantonal hospital St. Gallen Kantonsspital St. Gallen, St. Gallen, Switzerland; ||Cantonal Hospital Winterthur, Kantonsspital Winterthur, Winterthur, Switzerland; ${ }^{*}$ University Hospital Giessen and Marburg, Universitätsklinikum Giessen und Marburg GmbH, Giessen, Germany; $\dagger$ †City Hospital Triemli Stadtspital Triemli, Zürich, Switzerland; †tUniversity Hospital Düsseldorf, Dusseldorf Germany; §§University Hospital Lille Centre Hospitalier Régional Universitaire de Lille, Lille Cedex, France; $\boldsymbol{\top}^{\top}$ Cantonal hospital Olten, Olten, Switzerland Kantonsspital Olten, Switzerland, now at chirurgieaarau, Aarau, Switzerland; ||||Lausanne University Hospital, Lausanne, Switzerland; ${ }^{* *}$ Elisabethinen Hospital Linz Krankenhaus der Elisabethinen Linz, Linz, Austria; †††Eberhard Karls University Tübingen, Tübingen, Germany; 拉University Hospital Bern Inselspital Bern, Bern, Switzerland; $\S \S \S$ Cantonal Hospital Graubunden Kantonsspital Graubünden, Chur, Switzerland; $\bullet-$ Kliniken Essen-Mitte, Essen, Germany; and ||l|||Cantonal Hospital St. Gallen, St. Gallen Kantonsspital St. Gallen, Switzerland, now at Brustzentrum Ostschweiz, St. Gallen, Switzerland.

凶uvonholzen@goshenhealth.com.

This study was supported by research agreements with the following institutions: State Secretary for Education, Research and Innovation (SERI), Swiss Cancer Research Foundation (SCS), Swiss Cancer League (SCL) and a grant by the Swiss Foundation of Clinical Research and Merck KGaA, Darmstadt, Germany.

This study was presented at the Annual Meeting of the Society of Surgical Oncology in Chicago in March 2018.

The authors report no conflicts of interest.

Copyright (C) 2020 Wolters Kluwer Health, Inc. All rights reserved.

ISSN: 0003-4932/16/XXXX-0001

DOI: $10.1097 /$ SLA.0000000000004334 arm received adjuvant cetuximab. Surgical outcomes and complications were prospectively recorded and analyzed.

Results: Total of 259 patients underwent esophagectomy. Overall complication rate was $56 \%$ and reoperation rate was $15 \%$ with no difference in complication rates for transthoracic versus transhiatal resections ( $56 \%$ vs $54 \%, P=0.77$ ), nor for video assisted thoracic surgeries (VATS) versus open transthoracic resections $(67 \%$ vs $55 \%, P=0.32$ ). There was a trend to higher overall complication rates in squamous cell carcinoma versus adenocarcinoma (65\% vs $51 \%, P=$ 0.035 ), and a significant difference in ARDS in squamous cell carcinoma with $14 \%$ versus $2 \%$ in adenocarcinoma $(P=0.0002)$. For patients with involved lymph nodes, a lymph node ratio of $\geq 0.1$ was an independent predictor of PFS (HR 2.5, $P=0.01$ ) and OS (HR 2.2, $P=0.03$ ).

Conclusions: This trial showed no difference in surgical complication rates between transthoracic and transhiatal resections. For patients with involved lymph nodes, lymph node ratio was an independent predictor of progression free survival and overall survival.

Keywords: surgical outcomes after esophageal resection

(Ann Surg 2020;xx:xxx-xxx)

E sophageal cancer is the ninth most common cancer worldwide, - with about 572,034 new cases (3.2\% of all sites) estimated in 2018 , but the sixth leading cause of cancer related mortality with about 508,585 (5.3\% of all sites) estimated deaths in $2018 .{ }^{1}$ Multimodality treatment much improved overall survival rates for patients with resectable disease. ${ }^{2}$ However, esophageal cancer remains a highly fatal disease with a 5-year overall survival rate of around $20 \%$. Therefore, new treatment regiments are urgently needed. The Swiss Group for Clinical Cancer Research (SAKK) trial 75/08 was a multicenter phase III trial (NCT01107639) which investigated the addition of the EGFR-antibody cetuximab to a regimen of induction chemotherapy followed by chemoradiation and surgery for locally advanced esophageal cancer. Design and primary results of this study have been reported before. ${ }^{3}$ In brief, patients in the control arm received 2 cycles of induction chemotherapy consisting of cisplatin and docetaxel, followed by concomitant chemoradiation therapy with cisplatin, docetaxel and $45 \mathrm{~Gy}$. In the experimental arm, the same regimen was used with addition of cetuximab. After completion of neoadjuvant treatment, patients proceeded to esophagectomy. 
Patients in the experimental arm received cetuximab after surgery as adjuvant therapy in a 2-weekly manner for 6 cycles.

While there are a multitude of studies focusing on the surgical aspects, many basic treatment principles remain controversial, such as for example the optimal number of lymph nodes to be resected or the appropriateness of oncologic aspects for minimally invasive procedures and robotic procedures. Most controlled trials investigating surgical aspects of esophagectomy included low numbers of patients, therefore this randomized phase-1ll trial offered a great opportunity to assess the impact of surgical technique in regard to morbidity and mortality in the setting of an international, multicenter trial after neoadjuvant multimodal treatment.

\section{METHODS}

\section{Clinical Trial}

The SAKK trial 75/08 was an intergroup, open-label phase III trial performed according to declaration of Helsinki and GCP guidelines (NCT01107639). The protocol was approved by the ethics committee of each participating institution, and all patients gave written informed consent.

Design and primary results of this study have been reported before. $^{3}$ Briefly, previously untreated patients with resectable, locally advanced, histologically confirmed squamous cell carcinoma or adenocarcinoma of the thoracic esophagus, including the gastroesophageal junction (Siewert types I and II) were included.

Pretreatment staging consisted of complete medical history, physical examination, upper endoscopy with biopsy, Positron Emission Tomography - Computed Tomography (PET-CT) scan, computed tomography scans of the chest and abdomen, endoscopic ultrasound and bronchoscopy in case of tumors at or above the tracheal bifurcation.

In the control arm $\mathrm{B}$, the preoperative treatment consisted of 2 cycles of induction chemotherapy followed by chemoradiation. Induction chemotherapy consisted of cisplatin $75 \mathrm{mg} / \mathrm{m}^{2}$ and docetaxel $75 \mathrm{mg} /$ $\mathrm{m}^{2}$ administered i.v. on days 1 and 22. Chemoradiation started after the second induction chemotherapy cycle. Three-dimensional conformal radiotherapy of $45 \mathrm{~Gy}$ ( 25 fractions of $1.8 \mathrm{~Gy}$ ) was given over 5 weeks with 6 to $18 \mathrm{MV}$ photons. Concomitant chemotherapy consisted of i.v. cisplatin $25 \mathrm{mg} / \mathrm{m}^{2}$ and docetaxel $20 \mathrm{mg} / \mathrm{m}^{2}$, administered weekly for 5 weeks on an outpatient basis. The desired interval for esophagectomy in this study was within 4 to 7 weeks after administration of the last dose of radiation. This time frame was chosen because of our experience from earlier trials, and the very different rules about the timepoint of surgery after neoadjuvant chemoradiation in different centers in Europe. Despite the size of this trial including the international participation of 53 centers in 4 European countries, only 9 cases had delayed surgery. Seven cases were delayed due to logistical reasons, in the remaining 2 cases the reason for delay remained unspecified.

In the experimental arm A the same neoadjuvant schedule according to chemo- and radiotherapy was applied. In addition, cetuximab $250 \mathrm{mg} / \mathrm{m}^{2}$ was weekly administered during the whole chemotherapy (6 times) and chemoradiotherapy (5 times) with a loading dose on $\mathrm{d} 1$ of $400 \mathrm{mg} / \mathrm{m}^{2}$. Within 3 to 6 weeks (as early as possible) after surgery, cetuximab as adjuvant therapy was started in a 2-weekly manner with $500 \mathrm{mg} / \mathrm{m}^{2}$.

Every case of death under therapy, or until 90 days postoperatively, triggered immediate subsequent evaluation by a medical reviewer of SAKK together with the treating site to determine the exact reason for death. Every death was classified as not-treatmentrelated, direct or indirect complication of the treatment.

Surgical data and complications were assessed based on predefined lists. Chart review and queries were performed to capture missing data. Lymph node ratio was calculated as number of positive lymph nodes divided by total number of harvested lymph nodes.

\section{Statistics}

Categorical data were summarized using frequency counts and percentages and compared between subgroups using Fisher exact test. Time-to-event endpoints were summarized by the median and corresponding 95\% confidence interval using the Kaplan-Meier method. Effects of preselected covariates on these endpoints were explored using Cox regression models.

Two-tailed tests with significance level 0.05 were used for all analyses with no adjustment for multiple testing. All analyses were conducted using SAS 9.4 (SAS Institute Inc) or R 3.4.3 (www.rproject.org).

\section{RESULTS}

\section{Population and Tumor Characteristics}

A total of 300 patients with histologically confirmed esophageal squamous cell carcinoma or adenocarcinoma of the thoracic esophagus or the gastroesophageal junction (Siewert I and II) were randomized into the investigational arm or the control arm. Of these patients, 259 successfully underwent surgical resection. Baseline characteristics of these 259 patients undergoing surgical resection are shown in Table 1. An abbreviated CONSORT diagram is shown in Figure 1 . The majority of the patients was male. Sex, age, and weight was well balanced between the 2 groups. The median follow-up of the patients in this trial was 4 years. All of the patients eligible for this trial had a World Health Organization (WHO) performance status of $\leq 1$, adequate hematologic, renal and hepatic function and normal lung function, otherwise they could not be included.

\section{Surgery}

According to the protocol, esophageal en-bloc resection was recommended by an abdomino-thoracal or a thoraco-abdomino-cervical approach for squamous cell carcinoma with a complete two-field lymphadenectomy. Routine reconstruction was done by a gastric tube. For adenocarcinoma of the gastro-esophageal junction, Siewert type I, a subtotal esophagectomy was the preferred operation. For adenocarcinoma of the gastro-esophageal junction, Siewert type II, a transhiatal partial esophagectomy, including total gastrectomy with adequate lymphadenectomy and transhiatal esophagojejunostomy was recommended. The transhiatal approach in this study was chosen based on surgeon's preferences at that time in the participating centers in those 4 countries

In $87.3 \%(n=262)$ of the 300 patients analyzed, surgery was performed. $12.7 \%(\mathrm{n}=38)$ patients did not undergo surgery because of patient refusal in $3 \%(n=9)$, progressive disease or extent of disease in $3 \%(n=9)$, comorbidities in $2 \%(n=6)$, performance status in $1.7 \%(n$ $=5)$, death during or after neoadjuvant treatment in $1.3 \%(\mathrm{n}=4)$, trial stop due to unacceptable toxicity during neoadjuvant treatment in $1 \%$ $(\mathrm{n}=3)$ and ineligibility in $0.7 \%(\mathrm{n}=2)$. Of the patients that did undergo surgery, 3 did not undergo resection due to the extent of disease intraoperatively. Of the 259 resected patients, R0 Resection was achieved in $96.1 \%(\mathrm{n}=249)$ with no difference between the treatment groups. The rate of margin positivity in Siewert I and II tumors was $1.2 \%(\mathrm{n}=1)$ and $5.8 \%(\mathrm{n}=3)$, respectively.

Overall, the transthoracic approach was chosen in $75.3 \%(\mathrm{n}=$ $195)$, and the transhiatal approach in $23.6 \%(\mathrm{n}=61)$. The approach chosen differed based on the histology of the tumor. For adenocarcinoma, $69.0 \%(n=118)$ of procedures were performed transthoracic, and $29.2 \%(\mathrm{n}=50)$ transhiatal, versus for squamous cell carcinoma, $87.5 \%(\mathrm{n}=77)$ of the procedures were performed transthoracic, and only $12.5 \%(\mathrm{n}=11)$ transhiatal.

Of all the procedures that were performed with a transthoracic approach, $15.4 \%(\mathrm{n}=30)$ were performed as VATS.

According to the approach chosen, the level of anastomosis was placed thoracal in $67.6 \%(\mathrm{n}=175)$ and cervical in $31.7 \%$ 
TABLE 1. Baseline Patient Characteristics of Patients Undergoing Surgical Resection

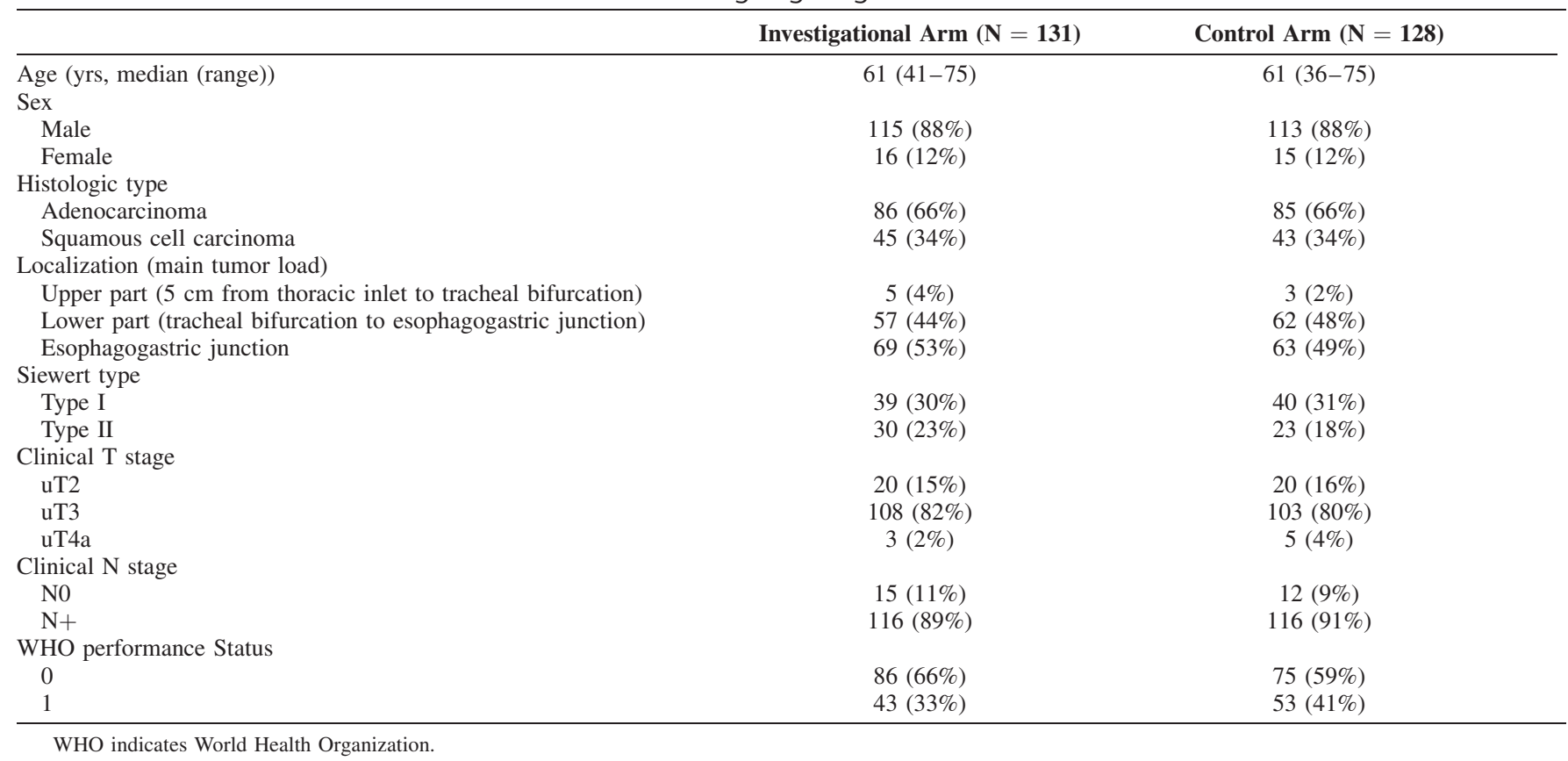

$(\mathrm{n}=82)$. Again, this slightly differed based on the histology. For adenocarcinoma $28.1 \%(\mathrm{n}=48)$ were cervical, and $70.8 \%(\mathrm{n}=121)$ thoracal, whereas for squamous cell carcinoma $38.6 \%(n=34)$ were cervical and $61.4 \%(\mathrm{n}=54)$ thoracal.

Surgical data according to histology is summarized in Table 2. There was no difference observed with the addition of cetuximab.

\section{Postoperative Complications}

The overall complication rate in this trial was $55.6 \%(\mathrm{n}=$ $144)$, with a reoperation rate of $15.4 \%(n=40)$ (calculated as percentage of patients having postoperative complications or reoperations, resp.). Stents were rarely placed (only $0.8 \% \mathrm{n}=2$ ). There was no difference in overall complication rates between the investigational $\operatorname{arm}(52.7 \% \mathrm{n}=69)$ and the control $\operatorname{arm}(58.6 \% \mathrm{n}=75)$, nor was there any difference in the reoperation rate $(16 \%(\mathrm{n}=21)$ versus $14.8 \%(\mathrm{n}=18)$, respectively).

The major complications in this trial were infections in $36.7 \%$ $(\mathrm{n}=95)$, with pneumonia most common with $23.2 \%(\mathrm{n}=60)$, anastomotic leak in $17 \%(\mathrm{n}=44)$ and Acute respiratory distress syndrome (ARDS) in 5.8\% $(\mathrm{n}=15)$ of the patients. There was a trend to higher overall complication rates and reoperation rates in squamous cell carcinoma versus adenocarcinoma $(64.8 \%(\mathrm{n}=57)$ versus $50.9 \%(\mathrm{n}=87), P=0.035$, and $18.2 \%(\mathrm{n}=16)$ versus $14.0 \%(\mathrm{n}=$ $24), P=0.468$, respectively). However this did not reach statistical

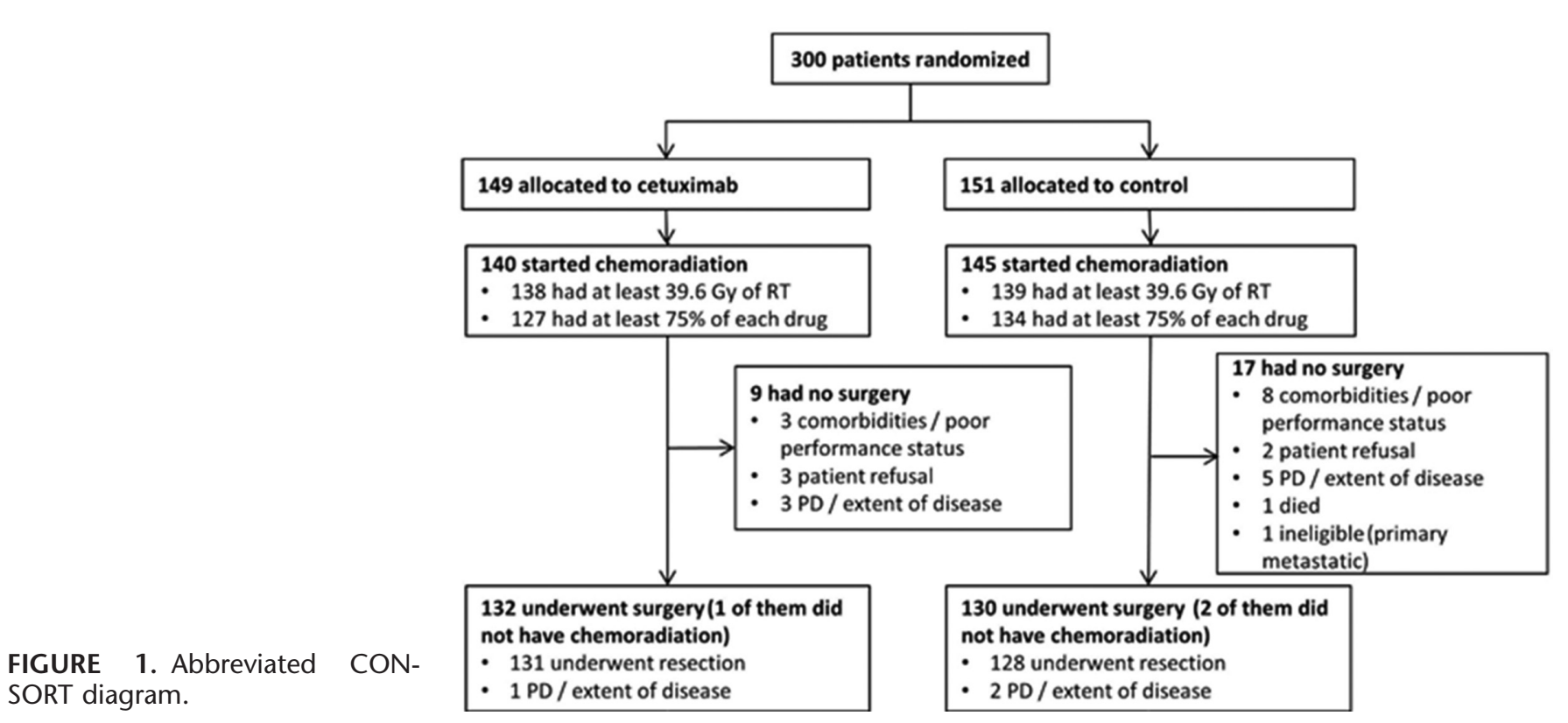


TABLE 2. Surgical Data Per Histology

\begin{tabular}{lcc}
\hline & $\begin{array}{c}\text { Adenocarcinoma } \\
(\mathbf{N}=\mathbf{1 7 1}) \\
\mathbf{n}(\boldsymbol{\%})\end{array}$ & $\begin{array}{c}\text { Squamous Cell } \\
\text { Carcinoma } \\
(\mathbf{N}=\mathbf{8 8}) \\
\mathbf{n}(\boldsymbol{\%})\end{array}$ \\
\hline $\begin{array}{l}\text { Resection } \\
\text { R0 }\end{array}$ & $165(96.5 \%)$ & $84(95.5 \%)$ \\
R1 & $5(2.9 \%)$ & $4(4.5 \%)$ \\
Localization of residual tumor & & \\
$\quad \begin{array}{l}\text { Distal/gastric margin } \\
\text { Proximal margin }\end{array}$ & $1(0.6 \%)$ & \\
$\quad$ Radial margin & $2(1.2 \%)$ & $4(4.5 \%)$ \\
Approach & $3(1.8 \%)$ & \\
$\quad$ Transthoracic & $118(69.0 \%)$ & $77(87.5 \%)$ \\
$\quad$ Transhiatal & $50(29.2 \%)$ & $11(12.5 \%)$ \\
For transthoracic approach & $100(58.5 \%)$ & $66(75.0 \%)$ \\
$\quad \begin{array}{l}\text { Open } \\
\text { Thoracoscopically (VATS) }\end{array}$ & $19(11.1 \%)$ & $11(12.5 \%)$ \\
Level of anastomosis & & \\
$\quad$ Cervical & $48(28.1 \%)$ & $34(38.6 \%)$ \\
$\quad$ Thoracic & $121(70.8 \%)$ & $54(61.4 \%)$ \\
\hline
\end{tabular}

significance. Important to mention is a significant difference in ARDS in squamous cell carcinoma with $13.6 \%(\mathrm{n}=12)$ versus only $1.8 \%(\mathrm{n}=3)$ in adenocarcinoma $(P=0.0002)$. Furthermore, pneumonia was also more frequent in squamous cell carcinoma than in adenocarcinoma [31.8\% $(\mathrm{n}=28)$ and $18.7 \%(\mathrm{n}=32)$, resp.]. Anastomotic leak rate was higher in squamous cell carcinomas as well $[22.7 \%(\mathrm{n}=20)$ vs $14.0 \%(\mathrm{n}=24)]$. ARDS was correlated with anastomotic leak and this correlation was statistically highly significant. ARDS occurred in $15.9 \%(\mathrm{n}=7)$ of patients with anastomotic leak versus $3.7 \%(\mathrm{n}=8)$ in patients without leak $(P=0.006)$. The same held true for postoperative pneumonia. There was an increased rate of pneumonia for patients having an anastomotic leak with $40.9 \%(\mathrm{n}=18)$ versus $19.5 \%(\mathrm{n}=42), P=0.005$. As expected, the overall complication rate was higher with resection of other organs [61.8\% ( $\mathrm{n}=47)$ vs $53.0 \%(\mathrm{n}=97), P=0.22]$.

There was no difference in complication rates for transthoracic versus transhiatal operations $[56.4 \%(\mathrm{n}=110)$ vs $54.1 \%(\mathrm{n}=33), P$ $=0.77]$, nor was there a difference in reoperation rates for transthoracic versus transhiatal operations $[16.9 \%(\mathrm{n}=33)$ vs $11.5 \%(\mathrm{n}=7)$, $P=0.42]$. For the transthoracic approach, complication rates were

TABLE 3. Major Postoperative Complication Rates Based on Histology and Surgical Approach

\begin{tabular}{|c|c|c|c|c|}
\hline & \multicolumn{2}{|c|}{$\begin{array}{c}\text { Adenocarcinoma }(\mathrm{N}=171) \\
\mathbf{n}(\%)\end{array}$} & $\begin{array}{c}\text { Squamous Cell Carcinoma }(\mathbf{N}=\mathbf{8 8}) \\
\text { n }(\%)\end{array}$ & $\begin{array}{c}\text { Total }(\mathrm{N}=259) \\
\text { n }(\%)\end{array}$ \\
\hline Infection & & $61(35.7 \%)$ & $34(38.6 \%)$ & $95(36.7 \%)$ \\
\hline C. diff colitis & & $2(1.2 \%)$ & $1(1.1 \%)$ & $3(1.2 \%)$ \\
\hline Empyema & & $9(5.3 \%)$ & & $9(3.5 \%)$ \\
\hline Line infection & & $2(1.2 \%)$ & & $2(0.8 \%)$ \\
\hline Peritonitis & & $1(0.6 \%)$ & & $1(0.4 \%)$ \\
\hline Pneumonia & & $32(18.7 \%)$ & $28(31.8 \%)$ & $60(23.2 \%)$ \\
\hline Port infection & & $1(0.6 \%)$ & & $1(0.4 \%)$ \\
\hline SIRS/Sepsis & & $7(4.1 \%)$ & $5(5.7 \%)$ & $12(4.6 \%)$ \\
\hline Urinary tract infection & & $4(2.3 \%)$ & $2(2.3 \%)$ & $6(2.3 \%)$ \\
\hline Wound infection & & $4(2.3 \%)$ & $2(2.3 \%)$ & $6(2.3 \%)$ \\
\hline ARDS & & $3(1.8 \%)$ & $12(13.6 \%)$ & $15(5.8 \%)$ \\
\hline Infection & & $67(34.4 \%)$ & $27(44$ & \\
\hline \multicolumn{5}{|c|}{ Specify infection (more than 1 applicable) } \\
\hline Abscess & & $2(1.0 \%)$ & $3(4$. & \\
\hline C. diff colitis & & $1(0.5 \%)$ & $2(3$. & \\
\hline Empyema & & $5(2.6 \%)$ & $4(6$. & \\
\hline Line infection & & $1(0.5 \%)$ & $1(1$. & \\
\hline Mediastinitis & & $5(2.6 \%)$ & & \\
\hline Pancreatitis & & $1(0.5 \%)$ & & \\
\hline Peritonitis & & & $1(1$. & \\
\hline Pneumonia & & $44(22.6 \%)$ & $15(24$ & \\
\hline Port infection & & & $1(1$. & \\
\hline SIRS/Sepsis & & $10(5.1 \%)$ & $2(3$. & \\
\hline
\end{tabular}

Other complications include: aortesophageal fistula, bleeding, bronchesophageal fistula, cardiac arrest / atrial fibrillation, chylothorax, DVT, hepatic insufficiency, pneumothorax, pulmonary embolism, recurrens paresis, renal insufficiency, small bowel injury, splenic infarction, TIA/neuro, tracheal injury. 
TABLE 4. Treatment Related Mortality Based on Histology and Surgical Approach

\begin{tabular}{|c|c|c|c|c|}
\hline & \multicolumn{2}{|c|}{ Adenocarcinoma } & \multicolumn{2}{|c|}{ Squamous Cell Carcinoma } \\
\hline & $\mathbf{N}$ & $\overline{\text { Death n }(\%)}$ & $\mathbf{N}$ & Death $\mathrm{n}(\%)$ \\
\hline Postoperative total & 171 & $10(5.8 \%)$ & 88 & $5(5.7 \%)$ \\
\hline - In-hospital & 171 & $6(3.5 \%)$ & 88 & $3(3.4 \%)$ \\
\hline \multirow[t]{2}{*}{$-\leq 90 \mathrm{~d}$} & 171 & $7(4.1 \%)$ & 88 & $5(5.7 \%)$ \\
\hline & \multicolumn{2}{|c|}{ Transthoracal } & \multicolumn{2}{|c|}{ Transhiatal } \\
\hline - In-hospital & 195 & $7(3.6 \%)$ & 61 & $2(3.3 \%)$ \\
\hline$-\leq 30 \mathrm{~d}$ & 195 & $9(4.6 \%)$ & 61 & $2(3.3 \%)$ \\
\hline$-\overline{\leq} 90 \mathrm{~d}$ & 195 & $11(5.6 \%)$ & 61 & $1(1.6 \%)$ \\
\hline
\end{tabular}

similar for VATS versus open procedures $[66.7 \%(\mathrm{n}=20)$ vs $54.8 \%$ $(\mathrm{n}=91), P=0.32]$.

There was a trend to a higher overall complication rate for cervical versus thoracic anastomosis $(63.4 \%(\mathrm{n}=52)$ vs $52.6 \%(\mathrm{n}=$ 92), $P=0.11$ ). This difference was mainly due to the anastomotic leak rate. There was a significantly higher rate of anastomotic leakages for cervical versus thoracic anastomosis $[25.6 \%(\mathrm{n}=21)$ vs $13.1 \%(\mathrm{n}=23), P=0.02)$. According to the Esophageal Complications Consensus Group (ECCG) score of complications after esophagectomy, $45.5 \%(\mathrm{n}=20)$ of patients with an anastomotic leak underwent a reoperation, and could therefore be classified retrospectively as Type III anastomotic leak. The remaining 24 patients $(54.5 \%)$ were classified as a Type I/II leak according to the ECCG classification, as these types could not be separated based on the data collected. Major complications are summarized in Table 3 according to histology and surgical approach. The specific reasons for take back to the operating room depending on the presence of an anastomotic leak are shown in Table 5. As mentioned above, there was no statistical difference with the addition of cetuximab.

\section{Mortality}

In total, 15 out of the 259 patients who underwent surgical resection died in the postoperative period due to complications for an overall mortality rate of $6 \%$ with no significant difference between the investigational arm and the control arm. Table 4 shows the detailed postoperative in hospital, 30-day and 90-day mortality rates of this trial according to histology and surgical approach.

TABLE 5. Reoperations Based on Presence of Anastomotic Leak

\begin{tabular}{|c|c|c|}
\hline & $\begin{array}{c}\text { No Anastomotic } \\
\text { Leak }(\mathbf{N}=\mathbf{2 1 5}) \\
\mathbf{n}(\%)\end{array}$ & $\begin{array}{c}\text { Anastomotic } \\
\text { leak }(N=44) \\
n(\%)\end{array}$ \\
\hline No Reoperation & $195(90.7 \%)$ & $24(54.5 \%)$ \\
\hline Reoperation & $20(9.3 \%)$ & $20(45.5 \%)$ \\
\hline \multicolumn{3}{|l|}{ Type of reoperation } \\
\hline Drainage & $2(0.9 \%)$ & $1(2.3 \%)$ \\
\hline Endovac & & $1(2.3 \%)$ \\
\hline Laryngoplasty & $1(0.5 \%)$ & \\
\hline Other nonrelated procedure & & $2(4.5 \%)$ \\
\hline Revision & $16(7.4 \%)$ & $13(29.5 \%)$ \\
\hline Stent & & $2(4.5 \%)$ \\
\hline Wound revision & $1(0.5 \%)$ & $1(2.3 \%)$ \\
\hline
\end{tabular}

\section{Length of Stay}

The median length of stay was 21 days (range 10-211) for the transthoracic approach and 18 days (range 3-144) for the transhiatal approach $(P=0.17)$. For patients with a transthoracic approach, the median length of stay was 24.5 days (range 13-211) for VATS procedures, and 20 days (range $10-170)$ for open procedures $(P=0.1)$.

\section{Lymph Node Resected, Lymph Node Ratio and Prognosis}

Median number of lymph nodes resected was 19.0 for the transthoracic and 18.0 for the transhiatal approach. In total $32 \%(\mathrm{n}=$ $83)$ of the patients had involved lymph nodes, with $29.0 \%(n=38)$ of the patients in the investigational arm, and $35.2 \%(\mathrm{n}=45)$ of patients in the control arm.

The total number of involved lymph nodes was a predictor of progression free survival (PFS) [hayard ratio (HR) $1.0695 \%$ CI (1.02$1.10), P=0.004]$, overall survival (OS) (HR 1.07 95\% CI [1.02-1.11], $P=0.003)$, time to locoregional failure $[\mathrm{HR}=1.0895 \% \mathrm{CI}(1.03-$ $1.14), P=0.003]$ and time to distant failure $[\mathrm{HR}=1.0795 \% \mathrm{CI}(1.01-$ 1.12), $P=0.01]$ in univariable Cox regression models.

For patients with involved lymph nodes, lymph node ratio of $\geq 0.1$ versus $<0.1$ was an independent predictor of PFS [HR $2.595 \%$ $\overline{\mathrm{CI}}(1.2-5.1), P=0.01]$, OS [HR 2.2 $95 \% \mathrm{CI}(1.1-4.4), P=0.03]$, time to locoregional failure [HR 4.1 95\% CI $(1.3-13.7), P=0.02]$ and time to distant failure [HR $2.995 \% \mathrm{CI}(1.1-7.4), P=0.03$ ] [Fig. $2 \mathrm{~A}-\mathrm{D}$ ].

\section{Survival}

Overall survival data of this trial has been reported before. ${ }^{3}$ The median progression free survival was 35 months in the investigational arm versus 24 months in the control arm $(\mathrm{HR}=0.79, P=$ 0.13 ). The median overall survival was 61 months in the investigational arm versus 36 months in the control arm $(\mathrm{HR}=0.73, P=$ 0.055 ), with 4 -year survival rates of $56 \%$ versus $43 \%$, respectively.

\section{DISCUSSION}

In this study, we found that addition of cetuximab to neoadjuvant therapy for esophageal cancer is safe as demonstrated by an overall low postoperative mortality rate. The SAKK trial 75/08 was a large European multicenter phase III trial conducted in Switzerland, Germany, Austria and France, and most patients were operated in low to median case load hospitals. The patient cohort in this trial was comparable to other published series, ${ }^{2}$ with most patients having advanced disease stages as expected by the trial design. The total postoperative mortality rate of $6 \%$ was consistent with other contemporary published series for multicenter trials of multimodality 

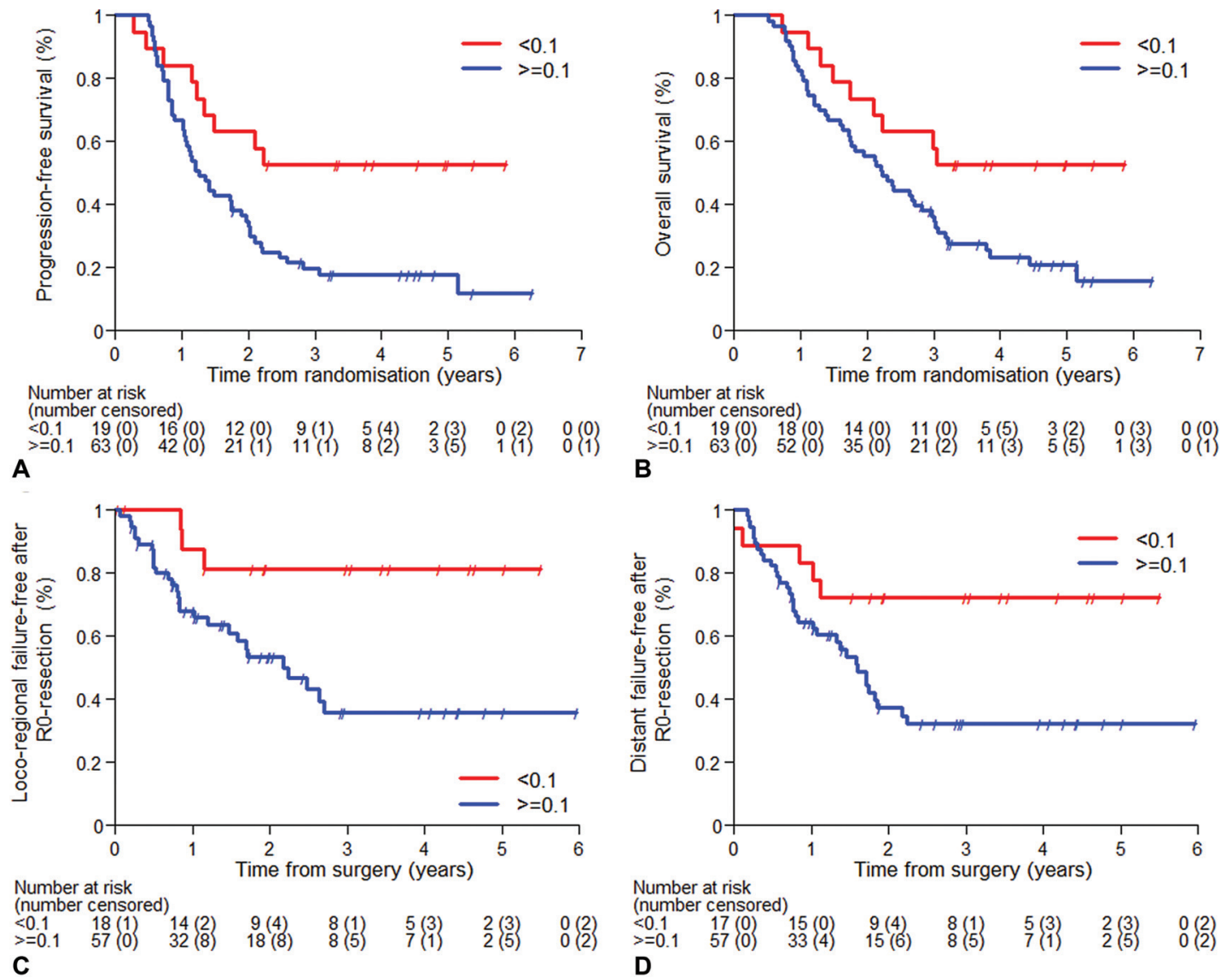

FIGURE 2. A. Kaplan-Meier-Plot for PFS by lymph node ratio (cut-off 0.1) for patients with involved lymph nodes. B. Kaplan-MeierPlot for OS by lymph node ratio (cut-off 0.1 ) for patients with involved lymph nodes. C. Kaplan-Meier-Plot for time to locoregional failure after R0 resection by lymph node ratio (cut-off 0.1 ) for patients with involved lymph nodes. D. Kaplan-Meier-Plot for time to distant failure after R0 resection by lymph node ratio (cut-off 0.1 ) for patients with involved lymph nodes.

treatment for esophageal cancer. ${ }^{4-6}$ Regarding overall and procedure specific complications, the observed rates were again consistent with those published in the literature. For example, a pulmonary complication rate of $45.2 \%$, an anastomotic leak rate of $26.0 \%$ and a cardiac complication rate of $18.9 \%$ were described in the CROSS trial. ${ }^{2}$ The overall complication rate of $55.6 \%$ in this study corresponds to the overall incidence of complications of $59.0 \%$ found in a recent publication of the ECCG involving 24 high-volume esophageal surgical centers in 14 countries. $^{7}$ Again, the good results in this study were achieved despite most of the patients being operated on in low to median case load hospital. Furthermore, in our trial, no difference was found regarding overall complication rates and outcomes between transthoracic and transhiatal esophageal resections, nor between adenocarcinomas and squamous cell carcinomas. However, there was a significant difference in the frequency of ARDS, with $13.6 \%$ in squamous cell carcinoma versus only $1.8 \%$ in adenocarcinoma $(P=0.0002)$. Furthermore, pneumonia was also more frequent in squamous cell carcinoma than in adenocarcinoma $(31.8 \%$ and $18.7 \%$, resp.). Possible explanations for this difference might be the generally higher location of the squamous cell carcinomas, placing more lung tissue into the neoadjuvant radiation field, and the location of the anastomosis, as ARDS was correlated with anastomotic leak. The same held true for postoperative pneumonia, where there was an increased rate of pneumonia for patients having an anastomotic leak. No difference was observed in reoperation rates for transthoracic versus transhiatal operations. Importantly, there was no difference in complication rates for minimally invasive versus open procedures. This was consistent with the emerging literature about the role of minimally invasive surgery in esophageal cancer. ${ }^{8,9}$ Regarding the site of the anastomosis, there was a trend to a higher overall complication rate for cervical versus thoracic anastomosis, but this failed to reach statistical significance. Most of the patients who have complications after esophagectomy have more than one complication. Unfortunately, at the conception of this study, the grading of the severity of surgical complications according to the Clavien Dindo classification was not included. Although a complete classification or even the comprehensive complication Index would be beneficial, we unfortunately do not have this data.

The median length of stay of 21 days was fairly long in this study compared to other published series. ${ }^{10}$ However, this might in part be explained by the traditions of the Health care systems in Switzerland, Germany, Austria and France where length of stay seems to be longer.

The number of lymph nodes that should be resected during esophagectomy remains controversial. Especially the effects of neoadjuvant chemoradiation therapy on lymph node retrieval during esophagectomy remain unclear. A recent study by Giugliano et al, comparing the quantitative lymph node assessment in patients after R0 
resection with or without neoadjuvant chemoradiation therapy, found that total lymph node counts were significantly lower in the chemoradiation therapy group, whereas positive lymph node counts and lymph node ratios did not differ from the upfront surgery group. The overall survival was decreased within the induction therapy cohort among those who had any positive lymph nodes retrieved at surgery. ${ }^{11}$ These results were consistent with the findings in our study. It is well known in the literature that the $\mathrm{N}$ status, or positive lymph nodes are an independent prognostic factor. In our study, the total number of involved lymph nodes was a predictor of progression free survival and overall survival in univariable Cox regression models. It has been shown previously that the number of involved lymph nodes can be used to predict the likelihood of systemic disease in patients with esophageal cancer, and thus survival of these patients. ${ }^{12}$ In our study, for patients with involved lymph nodes, a lymph node ratio of $\geq 0.1$ versus $<0.1$, and a lymph node ratio of $\geq 0.2$ versus $<0.2$, was an independent predictor of progression free survival and overall survival. Lymph node ratio has been found to have prognostic significance in several tumors. There are only a few reports in the literature that recognized the prognostic significance of the lymph node ratio in esophageal cancer, ${ }^{13,14}$ confirming our findings.

In conclusion, in a large European multicenter phase III trial, we found that addition of cetuximab to neoadjuvant therapy for esophageal cancer was safe as demonstrated by an overall low postoperative mortality rate and complication rate. There was no difference in postoperative complication rates regarding the surgical approach chosen. For patients with involved lymph nodes, lymph node ratio was an independent predictor of progression free survival and overall survival.

\section{REFERENCES}

1. Bray F, et al. Global cancer statistics 2018: GLOBOCAN estimates of incidence and mortality worldwide for 36 cancers in 185 countries. $C A$ Cancer J Clin. 2018;68:394-424.
2. Hagen Pv, Hulshof MCCM, Lanschot JJBv, et al. Preoperative chemoradiotherapy for esophageal or junctional cancer. N Engl J Med. 2012;366:20742084.

3. Ruhstaller T, Thuss-Patience P, Hayoz S, et al. Neoadjuvant chemotherapy followed by chemoradiation and surgery with and without cetuximab in patients with resectable esophageal cancer: a randomized, open-label, phase III trial (SAKK 75/08). Ann Oncol. 2018;29:1386-1393.

4. Steffen T, Dietrich D, Schnider A, et al. Recurrence patterns and long-term results after induction chemotherapy, chemoradiotherapy, and curative surgery in patients with locally advanced esophageal cancer. Ann Surg. 2019;269:83-87.

5. Fuchs HF, Harnsberger GR, Broderick RC, et al. Mortality after esophagectomy is heavily impacted by center volume: retrospective analysis of the Nationwide Inpatient Sample. Surg Endosc. 2017;31:2491-2497.

6. Markar S, Gronnier C, Duhame A, et al. Pattern of postoperative mortality after esophageal cancer resection according to center volume: results from a large European multicenter study. Ann Surg Oncol. 2015;22:2615-2623.

7. Low DE, Kuppusamy MK, Alderson D, et al. Benchmarking complications associated with esophagectomy. Ann Surg. 2019;269:291-298.

8. Biere SS, Henegouwen MIB, Maas KW, et al. Minimally invasive versus open oesophagectomy for patients with oesophageal cancer: a multicentre, openlabel, randomised controlled trial. Lancet. 2012;379:1887-1892.

9. van den Berg JW, Luketich JD, Cheong E. Oesophagectomy: the expanding role of minimally invasive surgery in oesophageal cancer. Best Pract Res Clin Gastroenterol. 2018;36-37:75-80.

10. Kauppila JH, Helminen O, Kytö V, et al. Short-term outcomes following minimally invasive and open esophagectomy: a population-based study from Finland and Sweden. 2018;25:326-332.

11. Giugliano DN, Berger AC, Pucci MJ, et al. Comparative quantitative lymph node assessment in localized esophageal cancer patients after R0 resection with and without neoadjuvant chemoradiation therapy. J Gastrointest Surg. 2017;21:1377-1384.

12. Peyre CG, Hagen JA, DeMeester SR, et al. Predicting systemic disease in patients with esophageal cancer after esophagectomy: a multinational study on the significance of the number of involved lymph nodes. Ann Surg. 2008;248:979-985.

13. Wang N, Rosato EL, Chojnacki KA, et al. Prognostic significance of lymph node ratio in esophageal cancer. Tumour Biol. 2015;36:2335-2341.

14. Tan Z, Ma G, Yang Y, et al. Can lymph node ratio replace pn categories in the tumor-node-metastasis classification system for esophageal cancer? J Thorac Oncol. 2014;9:1214-1221. 